\title{
A simplified in vitro ligation approach to clone an EIB55k-deleted double-targeted conditionally-replicative adenovirus Yosef S Haviv
}

Address: Department of Medicine, Hadassah-Hebrew University Medical Center, Jerusalem, 91120, Israel

Email: Yosef S Haviv - yhaviv@hadassah.org.il

Published: 7 February 2009

Virology Journal 2009, 6:18 doi:10.1186/1743-422X-6-18

This article is available from: http://www.virologyj.com/content/6/1/18

(C) 2009 Haviv; licensee BioMed Central Ltd.

This is an Open Access article distributed under the terms of the Creative Commons Attribution License (http://creativecommons.org/licenses/by/2.0), which permits unrestricted use, distribution, and reproduction in any medium, provided the original work is properly cited.
Received: 26 January 2009

Accepted: 7 February 2009

\begin{abstract}
Background: Construction of conditionally-replicative Adenovirus (CRAd) is complex and timeconsuming. While homologous recombination $(H R)$ using a two-plasmid system in bacteria is commonly used to generate CRAds, alternative methods may be required when HR fails. Previously, in vitro ligation has been suggested to facilitate construction of El/E3-deleted, replication-incompetent Ad vectors. However, in vitro ligation has only rarely been used to generate CRAds and may be a complex procedure for molecular biologists who are not experts in the field.
\end{abstract}

Methods and Results: A modified in vitro ligation approach was developed to construct a doubletargeted, EIB55k-deleted CRAd. The method allowed the incorporation of a tumor-specific promoter, e.g. the heat-shock protein 70 (hsp70) promoter, upstream of Ela, deletion of the EIB55k gene, and HR-free cloning of the recombined EI 55k gene into the Ad genome. The genetic structure of the CRAd was confirmed using restriction analysis and PCR. The replication rate of the hsp70E I $\triangle 55 \mathrm{k}$ CRAd was I.5-2\% of Ad without El $\Delta 55 \mathrm{k}$ deletion.

Conclusion: A 3-step cloning approach can generate a double-targeted, EIB55k-deleted CRAd using a straight-forward, modified in vitro ligation procedure.

\section{Introduction}

Adenoviruses (Ad) are excellent gene transfer vectors and are extensively used for high-level transgene expression in vitro and in vivo. One of the attributes rendering Ad vectors particularly well suited for this purpose is the capacity to manipulate their genome. Typically, Ad vectors are converted into mammalian gene transfer vectors by replacing the $E 1$ gene with the foreign gee of interest. E1 deletion serves two goals, i.e. to increase the cloning capacity to 5 $\mathrm{kb}$ (and to $8 \mathrm{~kb}$ if the $E 3$ region is also deleted) and to render Ad vectors replication-incompetent.

Two approaches to have been traditionally used to construct recombinant Ad vectors. First, homologous recom- bination (HR) in either packaging cell lines, bacteria or yeast. Second, in vitro ligation using standard molecular biology procedures. These two methods rely on the fact that purified, linear Ad DNA is infectious, resulting in recombinant Ad virions after transfection into packaging cell lines. In vitro ligation was the first approach to manipulate the Ad genome. However, it was abandoned for many years because the large, $35.9 \mathrm{~kb}$ Ad genome that encodes more than 50 gene products, generally lacks sufficient unique restriction sites. To overcome this limitation HR was developed, initially using ClaI digested viral DNA. Next, the viral DNA was transfected into the packaging cell line to undergo spontaneous, albeit inefficient HR. To enhance HR efficiency in mammalian cells, a two- 
plasmid rescue system was developed $[1,2]$. According to this method, two non-infectious plasmids with two overlapping Ad sequences were co-transfected into 293 cells, yielding an infectious recombinant Ad. The first use of the two plasmid system to construct recombinant Ad vectors incorporating foreign transgenes was developed by Graham's lab [3]. The manipulated plasmid encoding the transgene has been termed the shuttle plasmid and was co-transfected into 293 cells with along with a large plasmid containing the Ad backbone, incorporating a $2.2 \mathrm{~kb}$ ampicillin resistance gene in the XbaI site. Because neither plasmid is infectious alone, recombinant E1-deleted Ad vectors were generated as a result of HR between the overlapping regions. Further modifications were made by Bett who removed a large fragment from the E3 region and introduced a unique PacI site immediately adjacent to the Ad inverted terminal repeat (ITR), allowing linearization of the backbone plasmid [4]. HR thus employs two plasmids with overlapping sequences to be recombined in E1compelmenting 293 or 911 cells.

To construct Ad vectors encoding heterologous genes, the smaller plasmid, termed the shuttle plasmid and containing the left ITR, a packaging signal and a sequence overlapping with the larger Ad backbone plasmid, was engineered to encode the gene of interest. The larger plasmid, contained almost all the entire Ad genome devoid of the packaging signal and the E1 and E3 genes, was cotransfected into 293 cells where HR was to generate the recombinant Ad genome. However, this procedure required plaque purification of the recombinant Ad vector by screening individual clones. In addition, HR in 293 cells occurs in low frequency, is a time-consuming procedure and recombinant Ad vector progeny may be contaminated with the wild-type Ad virus. Thus, to obtain a pure Ad preparation at least two rounds of plaque purification assays may be required. To facilitate vector selection in cells, screening for the recombinant virus has been facilitated by using counter-selection methods, extensive fragmentation of Ad DNA complexed with the terminal protein or by using Cre-lox-mediated recombination [5]. These methods had the advantage that copies of the recombinant viral DNA were purified from clones and could therefore generate homogenous Ad preparations.

Optimization of two-plasmid HR was further reported in bacterial systems [6], using reconstitution of the sequence of the recombinant Ad in BJ5183 E. coli strains before transfection into 293 cells. Another advantage was preparation of the recombinant Ad genome in large quantities before transfection into 293 cells. The BJ5183 bacterial strain is recombination-proficient because i) it lacks the RecBCD enzyme and ii) it contains the RecF enzyme allowing DNA strand exchange between two linear DNA molecules that share at least 50 homologous bp at each end. One of these DNA molecules is the linearized shuttle plasmid previously subcloned with the heterologous gene and containing a bacterial origin of replication, a kanamycin-resistance gene and the left and right inverted terminal repeats (ITR) segments of the Ad genome.

While HR in bacteria has been generally accepted, it requires multiple steps in different $E$. coli stains. In addition, while in mammalian cells, the rate-limiting recombination step produced only the correct viral product of recombination, constructing the Ad genome by recombination in E. coli or yeast does not guarantee a correct, infectious recombinant Ad DNA. Unpredicted recombination events can occur, especially in yeast [7], requiring a thorough analysis of the recombinants to exclude clones that are unable to generate virus.

On this basis, in vitro ligation would be an attractive alternative procedure to HR. Until a decade ago, in vitro ligation was rarely used because of lack of unique restriction sites, low efficiency, the need for plaque purification to exclude wild-type Ad, and the risk of transgene-null $\mathrm{Ad}$ vectors due to self-religation. A major breakthrough in the methodology of in vitro ligation was the independent introduction of novel unique cleavage sites into the $\mathrm{Ad}$ genome by three independent groups [7-10]. Thus, in vitro ligation uses whole isolated Ad DNA cleaved with unique restriction sites flanking the E1-deleted region. The digested viral DNA is then ligated directly to a DNA fragment containing the left end joined to the gene of interest, followed by transfection of the recombinant Ad DNA into the packaging cell line [11]. One of the in vitro ligation techniques was developed by Mizuguchi and Kay via the introduction of unique intron-encoded endonuclease sites within the Ad genome [8,9] ('AdenoX ${ }^{\mathrm{Tw}}$ ). The I-CeuI/ PI-SceI intron-encoded endocnucleases uniquely cleave unusually long homing sequences ranging from 15 to 39 $\mathrm{bp}$, rendering these restriction sites rare and ideal for use as cloning sites in large genomes such as Ad. The E1deleted region is flanked by the two unique cleavage sites of I-CeuI and PI-SceI, and in between a SwaI cleavage site is used to inhibit re-ligation of the transgene-null Ad backbone. To produce E1-deleted, replication-deficient Ad vectors, this technique requires two cloning steps. First, subcloning the transgene into a I-CeuI/PI-SceI flanked, multicloning site (MCS) within the shuttle plasmid upstream of a CMV promoter and downstream of a poly A signal (Fig. 1d). Thus, cutting the recombinant shuttle plasmid with I-CeuI and PI-SceI provides a full expression cassette based on the foreign ORF CDNA inserted into the MCS. Second, cloning the recombinant I-CeuI/PI-SceI fragment from the shuttle vector into the I-CeuI/PI-SceI restriction site within the Ad backbone genome. Production of the recombinant Ad genome is thus truly in vitro and independent of bacterial or mammalian systems 

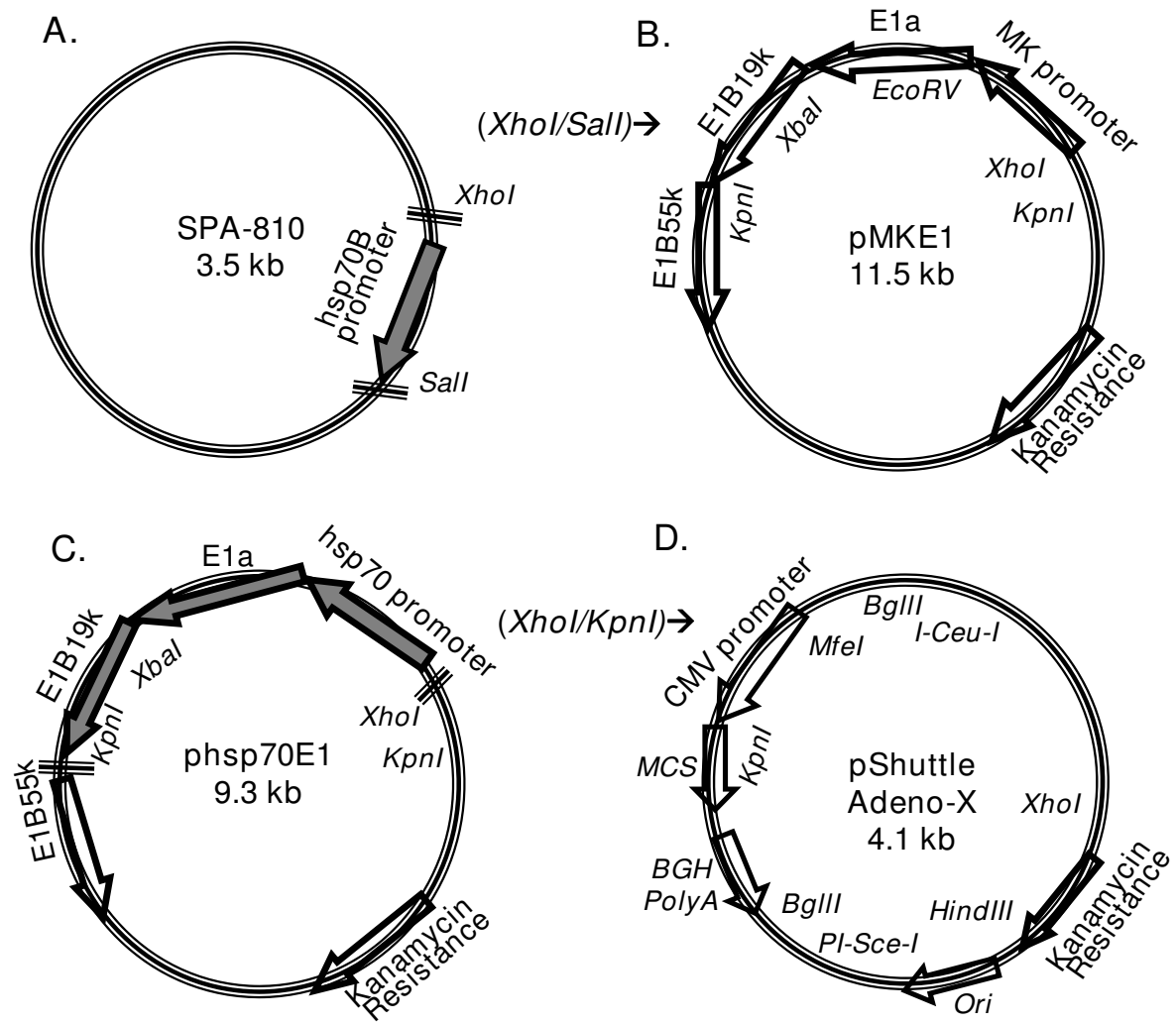

E.

F.

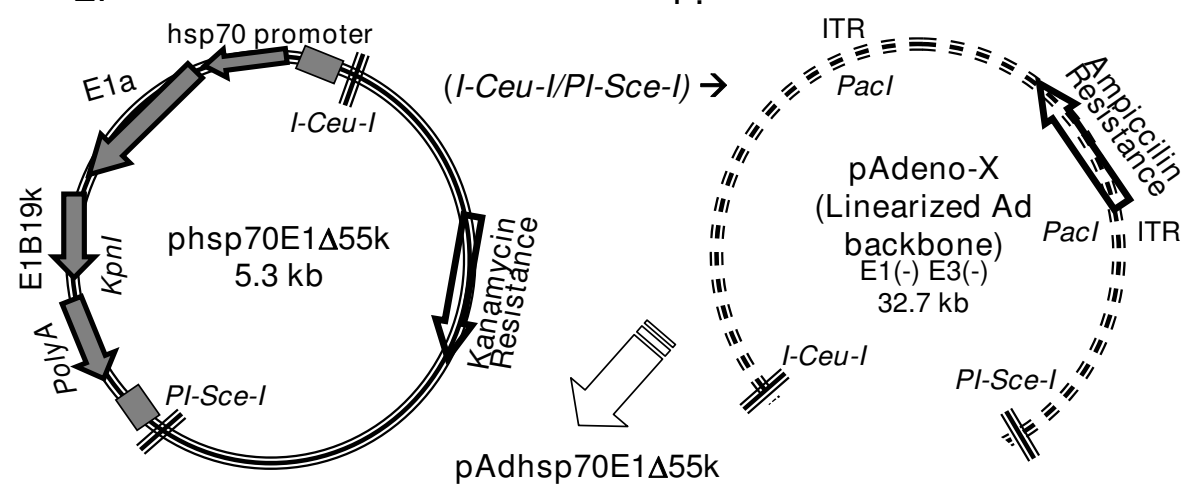

Figure I

Overall Schematic diagram of the 3 -step cloning strategy to generate CRAd-hsp70E I $\Delta 55 \mathrm{k}$ using a modified in vitro ligation system. To generate a double-targeted CRAd, a TSP-regulated EI $\triangle E I B 55 \mathrm{k}$ fragment was reintroduced into an EI/E3 deleted Ad backbone. a, the 383-bp human hsp70 promoter was cut with Sall, Klenow-dNTP blunted, Xhol digested, gel purified and inserted into the EcoRVIXhol site in PMKEI (b), to replace the MK promoter and generate phsp70EI (c). Next, to remove the EIB55k fragment, phsp70EI was digested with Xhol, Klenow dNTP blunted and digested with Kpnl (c). Simultaneously, to remove the CMV promoter yet maintain the polyA signal, pShuttle from the Adeno-X system was digested with $M$ fel, Klenow dNTP blunted and digested with Kpnl (d). Thus, the pShuttle of the AdenoX system was modified to encode a CMVpromoter deleted, TSP-regulated, EIB55k-deleted EI gene in phsp70EI $\Delta 55 \mathrm{k}(\mathbf{e})$. Next, to generate the recombinant CRAd genome, pAdhsp70EI $\Delta 55 \mathrm{k}$, the hsp70EI $\Delta 55 \mathrm{k}$ fragment was removed from phsp70EI with I-Ceul and PI-Sce-I digestion, purified via agarose gel electrophoresis and ligated into the pre-I-Ceul/PI-Sce-I-digested Ad backbone plasmid (f). Swal digestion eliminated the chance of religation of the Ad backbone plasmid without recombination. MK promoter, the human $2.6 \mathrm{~kb}$ gene promoter. CMV promoter, the human cytomegalovirus immediate-early gene promoter. BGH polyA, the bovine growth hormone early mRNA poly-adenylation signal. Grey-filled arrows indicate fragments excised for further cloning. 
encoding the enzymes essential for HR. However, because the shuttle plasmid contains a CMV promoter upstream of the MCS, tight control of transgene expression may be lost.

Similarly, Souza and Armentano employed the same logic for the construction of Ad serotype 2 vectors using I-CeuI and SnaBI sites [10]. However, their pAdvantage ${ }^{\mathrm{TM}}$ system has a substantially lower reported cloning efficiency than the method reported by Mizuguchi and Kay [8,9].

Danthinne also developed a HR-free system ('AdenoQuick $^{\mathrm{TMI}}$ ) for preparation of replication-deficient Ad, using a relatively complex system requiring encapsidation of the recombinant Ad genome into $\lambda$ phage particles [7].

In the context of oncolytic Ad, re-introduction of modified $E 1$ cassettes is required to construct conditionally-replicative Ad (CRAd). The superiority of CRAds over replication-deficient Ad vectors for cancer includes selective amplification of the cancer cell-killing capacity while relatively sparing normal cells. The attributes of CRAds include lysis of tumor cells that are resistant to standard therapy, selective cancer cell killing and induction of cellmediated anti-tumor immunity. Because there is currently no established effective therapy for disseminated Ad infection, CRAd replication should be stringently restricted to permissive cancer cells. To this end, two genetic approaches have been reported involving either insertion of a tumor-specific promoter (TSP) upstream of $E 1 a$ and $E 4$ or partial deletion of E1 genes, e.g. E1B55k or the E1A conserved region 2 (CR2) [12]. Infrequently, double-targeted CRAds may combine both types of genetic modifications.

In contrast to generation of replication-deficient Ad vectors, construction of CRAds is not straight-forward and may be time and labor-consuming. Two molecular strategies to construct CRAds have been described. First, and by far more popular, HR has been used by many groups to recombine an E1-encoding shuttle plasmid with the pAdEasy backbone plasmid [13-22]. The second strategy to generate CRAds involves in vitro ligation [23-28]. The setbacks of HR in the context of CRAds include the unpredicted chance of in vivo HR in BJ5183 bacteria and the lack of sufficient plasmid DNA production in BJ5183 bacteria required to allow diagnostic restriction analysis, thereby requiring transfer to candidate plasmids into other $E$. coli strains, such as DH5 $\alpha$ [29].

Despite the utility of HR, potentially targeting virtually the entire Ad genome, when HR fails, alternative approaches to construct CRAds may be required. The two reported approaches to generate CRAds using in vitro ligation differ in their methodologies [23-27]. First, Hernandez-
Alcoceba et al have reintroduced E1 $a$ and $E 4$ into the Ad genome $[23,24]$. To generate a CRAd, direct cloning of TSPs upstream of E1a or E4 within the Ad backbone is feasible using unique restriction sites flanking the promoter regions of E1A (BstBI sites) and E4 (I-CeuI and SwaI sites) $[23,24]$.

The setbacks of this approach may include the complete lack of the two E1B genes and the large Ad backbone containing the $E 1$ and $E 4$ genes, rendering manipulation of E1/E4 within the large Ad genome less efficient than in smaller shuttle plasmids which are readily amenable for genetic modifications.

The second in vitro ligation approach to generate CRAds without HR was developed by Danthinne [25-28]. This method is based on the AdenoQuick ${ }^{\mathrm{TM}}$ system, originally developed to construct replication-deficient E1-deleted Ad vectors [7]. To construct CRAds, this strategy involves re-introduction of modified E1 genes into a shuttle plasmid containing the Ad left arm and the Ad packaging signal. While several CRAds were reported using this system [25-28], the process is rather complex and may require multi-order fragment ligation $[25,27]$ and a cos site next to the gene of interest for subsequent in vitro packaging into phage $\lambda$ after cloning the recombinant Ad backbone [7]. Thus, this in vitro methodology for CRAd construction may be too labor and time-consuming to investigators who are not experts in the field.

In the current study we report a simplified, HR-free in vitro ligation approach to construct a double-targeted, TSP-regulated, $\triangle$ E1B55k CRAd involving a direct, 3-step cloning strategy. This modified in vitro ligation system is a simple and efficient method to construct recombinant $\Delta \mathrm{E} 1 \mathrm{~B} 55 \mathrm{k}$ CRAds.

\section{Results and discussion}

As a proof of principle to construct a double-targeted CRAd via in vitro ligation, CRAd-hsp70E1 $\Delta 55 \mathrm{k}$ was generated using two genetic modifications, e.g. cloning the heat-shock 70 promoter (hsp70) TSP upstream of the E1A gene and deletion of the E1B55kD gene (Fig. 1). Overall, we combined components from the AdEasy [6] and the Adeno-X $[8,9]$ systems. An E1-encoding plasmid, constructed on the basis of the pShuttle of the AdEasy system [14], was employed to subclone the hsp70 promoter upstream of E1 (Fig. 1b). However, instead of using this shuttle plasmid for HR, we further subcloned an E1B55kdeleted, TSP-regulated $E 1$ construct from this shuttle plasmid into the shuttle vector of the Adeno-X system (Fig. $1 \mathrm{~d})$. The modified $E 1$ construct was then directly cloned into an E1/E3 deleted Ad backbone genome using in vitro ligation instead of HR (Fig. 1e, f). 
The first step involved insertion of a TSP upstream of E1. The human hsp70 promoter was selected as a TSP because hsp70 expression has been associated with malignancy [30]. Furthermore, heat, ultrasound and magnetic resonance imaging (MRI)-inducible transcription may enable spatial and temporal control of hsp70-regulated gene expression [20,31,32]. Thus, regulation of $E 1 \mathrm{~A}$ by the hsp70 promoter may be a rational TSP approach in the context of CRAds. To insert the $383 \mathrm{bp} h s p 70 B$ promoter $(-270 \rightarrow+113)$ upstream of the E1A gene, the SPV-110 plasmid was digested by SalI, Klenow-dNTP blunted and cut with XhoI (Fig. 1a). Klenow treatment was required to blunt the overhang SalI end because the hsp70 promoter was inserted into an EcoRV/XhoI site within the pMKE1 (Figs. 1b and 2). pMKE1 was originally derived from the shuttle plasmid of the AdEasy system via sequential cloning of $E 1$ components into the multiple cloning site [14]. pMKE1 contains the complete E1 and protein IX genes and is deleted of the native E1 $a$ promoter $(\Delta 324-488 \mathrm{nt}$ from the left Ad arm). However, in contrast to the next step of HR in the AdEasy system, the XhoI/KpnI digested fragment, encompassing the hsp70 promoter, E1 $a$ and $E 1 B 19 k$, and deleted of the E1B55k gene by KpnI digestion, was directly subcloned into the pShuttle of the Adeno-X system (Fig. 1d). Within the latter pShuttle, the CMV promoter is retained to drive expression of the heterologous transgenes in replication-deficient Ad vectors $[8,9]$. However, because CRAds require a TSP upstream of $E 1 A$, the CMV promoter was excised via MfeI/KpnI digestion and replaced by the hsp70E1 $\Delta 55 \mathrm{k}$ construct, immediately flanked by the stop codons of the AdenoX pShuttle (Figs. 1c-e and 2).

Next, the I-Ceu-I/PI-Sce-I fragment was excised from phsp70E1 $\triangle 55 \mathrm{k}$ shuttle plasmid, and cloned into the ICeu-I/PI-Sce-I flanked, E1-deletion site within the Adeno$\mathrm{X}$ backbone plasmid to produce pAdhsp70E1 $\Delta 55 \mathrm{k}$ (Figs. $1 \mathrm{e}-\mathrm{f}, 3)$. Of note, because the E1-deleted Ad backbone pAdeno-X is also deleted of the E1a promoter (deleted from nt. 342 of the left arm of the Ad genome), this cloning approach results in a recombinant CRAd genome with TSP-regulated E1a expression. This ligation procedure resulted in the correct recombinant CRAd genome in 10\% of ampicillin-resistant colonies screened by restriction analysis and confirmed by PCR (Fig. 3). After large scale preparation of the recombinant plasmid with Qiagen Maxi-kit, the recombinant pAdhsp70E1 $\triangle 55 \mathrm{k}$ plasmid was linearized with PacI digestion and transfected into 293 cells to produce CRAd-hsp70E1 $\Delta 55 \mathrm{k}$ without the need for plaque purification. Absence of wild-type Ad contamination in CRAd-hsp70E1 $\Delta 55 \mathrm{k}$ stocks was confirmed after three viral passages by absence of E1B55k DNA detection via real-time PCR (Fig. 4a). Thus, a double-targeted CRAd was generated using straight-forward HR-free, in vitro ligation.
Of note, CRAd-hsp70E1 $\Delta 55 \mathrm{k}$ manifested a decreased cytopathic effect relative to AdCMVE1 (not shown), deriving from attenuated viral replication. The relative viral DNA replication rate of CRAd-hsp70E1 $\Delta 55 \mathrm{k}$ after 36 hours was $1.98 \%$ and $1.52 \%$ of Ad viruses driven by the CMV promoter (AdCMVE1) or the native E1a promoter (Ad5luc3), respectively (Fig. 4b). The fidelity of the hsp70 promoter in CRAd hsp70E1 $\Delta 55 \mathrm{k}$ was not maintained during heat induction, i.e. there was no heat-shock induction of CRAd hsp70E1 $555 \mathrm{k}$ DNA replication (not shown). This finding is in accordance with previous reports of hsp70 promoter in replicating Ad $[20,33]$. Thus, while heat-shock enhances the oncolytic effect of CRAds in general [34], the hsp70 promoter does not provide tight control in the context of CRAd-hsp70E1 $\Delta 55 \mathrm{k}$.

CRAd-hsp70E1 $\triangle 55 \mathrm{k}$ differs from two previously-reported CRAds with hsp70 promoters. The first hsp70 CRAd contained a $\Delta \mathrm{E} 1 \mathrm{~B} 55 \mathrm{k}$ deletion, but the hsp70 promoter did not drive E1 a expression but rather a cytosine deaminase/ thymidine kinase $(C D / T K)$ suicide gene [33]. The second hsp70 CRAd combined both hsp70 and mouse tyrosinase promoters to drive expression of $E 4$, without tumor-targeted partial E1a or E1B deletions [20]. Of note, both these hsp70-CRAds, as the vast majority of other CRAds, were produced using HR.

Deletion of the E1B55k gene was the genetic modification in the first CRAd, dl1520 (Onyx-015) [35]. The deletion of a viral gene such as $E 1 B 55 k$ that inactivates cellular regulatory proteins, e.g. p53, was suggested to restrict CRAd replication to cancer cells with specific genetic mutations thought to complement the viral genetic loss of function [35]. The Ad E1B gene encodes two major species of mRNAs. One mRNA codes for a 19-kDa polypeptide $(E 1 B 19 k)$ and the other codes for a $55-\mathrm{kDa}$ protein $(E 1 B 55 k)$. The two proteins are encoded by alternative reading frames and share no sequence homology. During Ad infection the E1B proteins inhibit apoptosis to allow Ad protein production and viral DNA replication. While the E1B19k protein has an anti-apoptotic effect mimicking the cellular $b c l-2$, The E1B55k protein exerts its antiapoptotic effect via inhibition of p53-induced transcription. In addition, E1B55K induces a cellular environment conductive for viral protein synthesis via a complex with the Ad E4 ORF6 protein. This complex inhibits the transport of host cellular mRNA from the nucleus to the cytoplasm while selectively stabilizing and transporting viral mRNA. Because approximately half of human cancer types are mutated for $p 53$, deletion of the E1B55k gene has been suggested as a tumor-targeted approach suggesting conditional Ad replication only in p53-mutant cells [35]. This approach has been subsequently disputed [36], but E1B55k deletion is still considered one of the genetic approaches to mitigate Ad replication in normal cells 
A
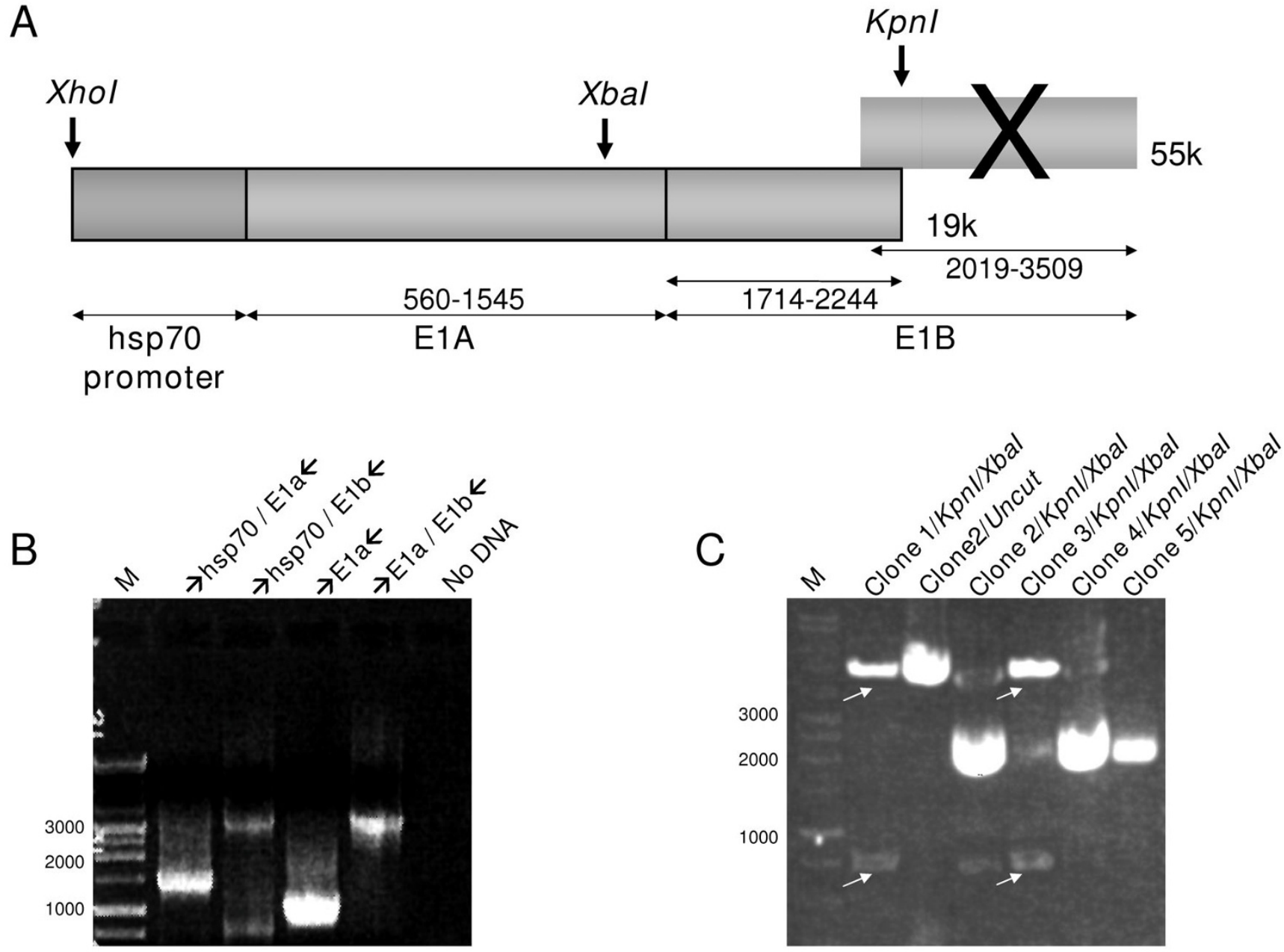

Figure 2

Validation of hsp70E I $\triangle 55 \mathrm{k}$ fragment. a. Schema of the recombinant hsp70EI $\triangle 55 \mathrm{k}$ cassette obtained by Xhol/Kpnl digestion of phsp70EI. b. The construct of phsp70EI (see Fig. Ic) was confirmed by PCR using complementary oligonucleotide amplimers of sequences flanking the indicated fragments of the template plasmid. c. Candidate clones for phsp70EI $\Delta 55 \mathrm{~K}$ (see Fig. (e) were screened using digestion analysis with $\mathrm{Kpnl}$ and $\mathrm{Xbal}$. Diagnostic fragments obtained are marked with an arrow. $\mathrm{M}$-The I kb DNA ladder from Gibco was used as DNA size marker.

[12], probably via loss of E1B55k-mediated late viral RNA export rather than p53-inactivation [36]. The cloning strategy reported here allows the deletion of E1B55k via digestion of the E1-encoding vectors pMKE1 or phsp70E1 at the $k p n I$ site. A prior step of subcloning a TSP upstream of the E1a gene allows the construction of a double-targeted $E 1$ gene for subsequent cloning into the Ad genome. Thus, in view of the relatively complex construction of CRAds using HR [13-22] and previously-reported in vitro ligation approaches [23-28], the method we propose has the advantage of only 3 standard cloning steps required to produce a double-targeted CRAd.

The drawbacks of this approach include limitation to E1encoding expression cassettes free of ClaI, SwaI and PacI, BstBI and Psp1406I sites. In addition, in vitro ligation is generally limited to recombining the ends of the Ad genome at the E1 and E4 regions, while HR can use linearized vectors to target virtually any region in the Ad backbone to allow point mutations and small deletions.

In conclusion, HR and in vitro ligation have been frequently and rarely employed to generate CRAds, respectively. The simplified in vitro ligation system described herein may be efficiently employed to construct TSPdriven, E1 55k-deleted CRAds.

\section{Materials and methods Cells, plasmids and reagents}

293 and A549 cells were from ATCC (Manassas, VA) and maintained in DMEM supplemented with $10 \%$ fetal calf serum, 100 units $/ \mathrm{ml}$ of penicillin and $100 \mu \mathrm{g} / \mathrm{ml}$ of strep- 


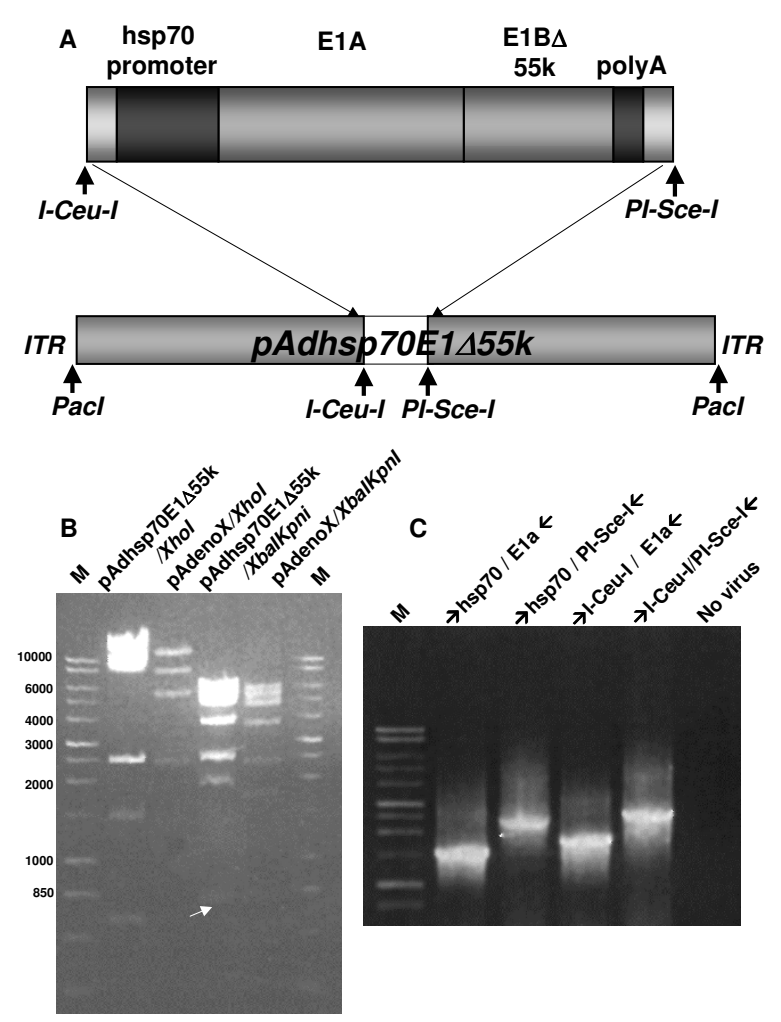

Figure 3

Validation of pAdhsp70EI $\Delta 55 \mathrm{k}$. a. Schema of the recombinant Ad plasmid pAdhsp70EI $\Delta 55 \mathrm{k}$. b. Restriction analysis to confirm the structure of pAdhsp70EI $\Delta 55 \mathrm{k}$ by $\mathrm{Xbal} / \mathrm{Kpnl}$ $E I$ cleavage. The diagnostic fragment is marked with an arrow. Control was the Adeno-X Ad backbone plasmid. c. Standard PCR was used to confirm the correct structure of the recombinant CRAd-Adhsp70EI $\Delta 55 \mathrm{k}$. The purified recombinant virus was directly applied in the various indicated $\mathrm{PCR}$ reactions encompassing the modified $\mathrm{EI}$ gene within the PI-Scel/I-Ceul site of the recombinant CRAd.

tomycin in 5\%CO2. The SPV-110 plasmid (StressGen, Victoria, Canada) contains the 383 bp human hsp70B promoter (GenBank accession no. X13229), flanked by XhoI and SalI restriction sites. pMKE1 was previously described [14]. Briefly, it was derived from the shuttle plasmid of the pAdeasy system where the multi-cloning site and right arm in the pAdeasy pShuttle were replaced by the $2.6 \mathrm{~kb}$ midkine $(\mathrm{MK})$ promoter and the complete Ad $E 1$ region and protein IX genes, devoid of the native Ad E1a promoter region $(\Delta 324-488)[14]$. pShuttle and pAdenoX backbone were from Clontech, San Jose, CA (currently $B D$, Franklin Lakes, NJ). All restriction enzymes and ligase were from New England BioLabs except for I-CeuI and PI-Sce-I (Clontech). All DNA purificaiotn and cell lysis kits were from Qiagen (Valencia, CA).

\section{Standard molecular biology procedures for in vitro CRAd construction}

Screening for recombinants was with miniprep plasmid DNA prepared by standard alkaline lysis kit (Qiagen), restriction analysis and $0.8 \%$ agarose gel electrophoresis with ethidium bromide staining. Large scale Ad backbone plasmid and recombinant Ad plasmids were purified by phenol-chloroform extraction and ethanol precipitation or Maxi-prep kit (Qiagen). Digested hsp70 promoter and hsp70E1 $\Delta 55 \mathrm{k}$ fragments were purified by gel extraction after agarose gel electrophorsesis. To clone the hsp70E1 $\Delta 55 \mathrm{k}$ fragment from phsp70E1 into pShuttle (Fig. 1c, d), a ratio of 3:1 (insert: digested pShuttle) was optimal. Ligation of the I-Ceu/I-PI-Sce-I flanked hsp70E1 $\Delta 55 \mathrm{k}$ fragment (after digesting $1 \mu \mathrm{g}$ of phsp70E1 $\Delta 55 \mathrm{k}$ for 3-hrs) into $750 \mathrm{ng}$ of the pAdeno-X backbone was performed with T4 DNA ligase overnight at $16^{\circ} \mathrm{C}$. Cloning efficiency was enhanced by heat inactivation of the T4 DNA ligase and by digesting with SwaI that recognizes a unique site between the I-Ceu-I/PI-Sce-I sites of the Adeno-X backbone (but not within the I-Ceu-I/PISce-I fragment from phsp70E1 $\Delta 55 \mathrm{k})$, thereby preventing religation of the E1-deleted backbone. The recombinant pAdhsp70E1 $\Delta 55 \mathrm{k}$ plasmid was used to transform $50 \mu \mathrm{l}$ chemical-competent DH5 $\alpha$ bacteria via 60 sec heat-shock and selection with ampicillin $(100 \mu \mathrm{g} / \mathrm{ml})$.

To rescue CRAd-hsp $70 \mathrm{E} 1 \Delta 55 \mathrm{k}, 5 \mu \mathrm{g}$ of pAdhsp70E1 $\Delta 55 \mathrm{k}$ were linearized with PacI to expose both ITRs and DNA was purified by phenol-chloroform. Next, to transfect 293 cells, $4 \mu \mathrm{g}$ of the digested DNA was mixed at room temperature with $20 \mu \mathrm{l}$ Lipofectamine (Life Technologies), diluted in $500 \mu \mathrm{l}$ of OptiMEM (Life) and added to the DMEM media for 4-hrs. Media was replaced to growth media and 293 cultured for 12 days until a cytopathic effect was observed. After three cycles of viral propagation, CRAd-hsp70E1 $\triangle 55 \mathrm{k}$ was purified using double CsCl density gradient centrifugation and tittered using both DNA optical density at $260 \mathrm{~nm}$ (viral particle [vp] concentration) and plaque assays in HEK 293 cells (infectious units [iu]). The vp/iu ratio was 100 .

\section{Validation of constructs using PCR}

The oligonucleotide primer corresponding to the human hsp70B promoter was sense strand 5'-AGCTAGAACCTTCCCCGCG-3'. The oligonucleotide primers corresponding to the Ad E1a were sense strand 5'-TCTTGAGTGCCAGCGAGTAG-3' and the antisense strand was 5'AAGTCCAAAGGTTGCCCAGG-3'. The oligonucleotide primers corresponding to the Ad E1B were sense strand 5'TTTTCTGCTGTGCGTAACTT-3' and the antisense strand was 5'-ATCTTCATCGCTAGAGCCAA-3. For whole E1 PCR the forward primer was the sense sequence of $E 1 a$ and the reverse prime was the antisense sequence of $E 1 B$. The oligonucleotide primer corresponding to the forward I-Ceu-I 

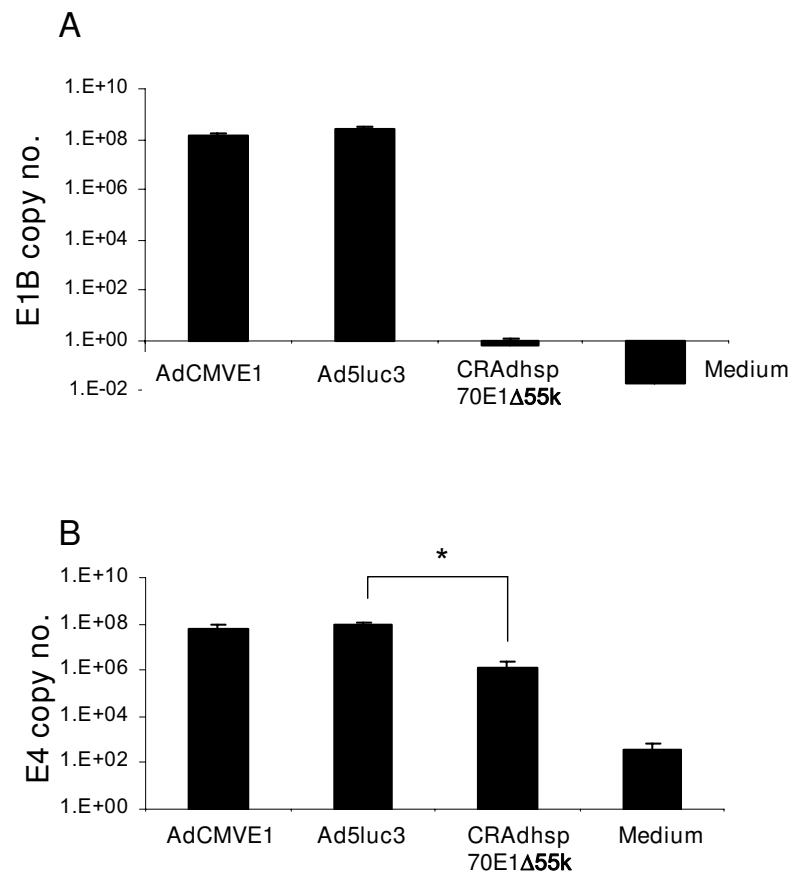

Figure 4

Analysis of CRAd-hsp70E I $\triangle 55 \mathrm{k}$ replication using real-time PCR. a, to confirm EIB55k deletion from CRAdhsp70E I $\triangle 55 \mathrm{k}$ and absence of wild-type Ad, I50,000 A549 cells were plated in I2-well plates and infected after 24-hrs in triplicates with $10 \mathrm{MOI}$ (iu) of CRAd hsp70E I $\Delta 55 \mathrm{k}$, AdCMVEI or Ad5luc3. Thirty-six hours later cellular DNA was extracted and real-time PCR was performed to measure the EIB gene copy number using primers encompassing the EIBI9k (forward primer) and EIB55k (reverse primer) sequences. b, to evaluate CRAd-hsp70E I $\triangle 55 \mathrm{k}$ replication relative to non-attenuated $A d$, real-time $P C R$ was performed on DNA extracted from cells infected above in (a), and the E4 gene copy number was measured. *, $p<0.05$ for attenuated CRAd-hsp70EI $\Delta 55 \mathrm{k}$ replication vs. Ad5luc3 ( $t$ test).

sense strand was 5'-TAACTATAACGGTCCTAAGGTA-'3 and the reverse PI-Sce-I primer was 5'-TTTCTCCGCACCCGACATAGA-'3. To analyze viral DNA with standard PCR, $1 \mu$ l of purified CRAd-hsp70E1 $\Delta 55 \mathrm{k}$ was directly applied into a $50 \mu \mathrm{l}$ PCR reaction with $1 \mu \mathrm{l}$ of each primer, at 30 cycles of $95^{\circ} \mathrm{C} 1$-min denaturation, $50^{\circ} \mathrm{C} \mathrm{2-min}$ annealing and $72^{\circ} \mathrm{C} 2.5$-min extension.

\section{Analysis of CRAd replication with real-time PCR}

One hundred and fifty thousand A549 cells were infected in triplicates with 10 MOI of CRAd hsp70E1 $555 \mathrm{k}$, AdCMVE1 or Ad5luc3. Thirty-six hours later (to allow one cycle of complete Ad DNA replication) cellular DNA was extracted using a Blood DNA purification kit (Qiagen). Real-time PCR conditions were 35 cycles of $\left(94^{\circ} \mathrm{C}, 20 \mathrm{~s} \rightarrow\right.$ $\left.55^{\circ} \mathrm{C}, 20 \mathrm{~s} \rightarrow 72^{\circ} \mathrm{C}, 30 \mathrm{~s}\right)$. Ad backbone plasmid pTG3602 (Transgene, Strasbourg, France) was available for plotting a standard curve for the E4 copy number. E4 copy numbers were normalized to the -actin DNA copy number.

One $\mu$ l of eluted DNA sample was analyzed by real-time PCR amplification to measure Ad E4 copy number as an indicator of CRAd replication and $E 1 B$ copy number to confirm E1B55k deletion in CRAd-hsp70E1 $555 \mathrm{k}$ and absence of wild type Ad contamination. The oligonucleotide primers corresponding to the $\mathrm{E} 1 \mathrm{~B}$ sense strand were 5'-GACAGGGCCTCTCAGATGCT-3' (Ad genome 30753094) and the antisense strand was 5'-5'-TGGCTACGTGAATGGTCTTCAG-3' (Ad genome 3144-3123). The oligonucleotide primers corresponding to the E4 sense strand were 5'-TGACACGCATACTCGGAGCTA-3' (Ad genome 34885-34905) and the antisense strand was 5'TTTGAGCAGCACCTTGCATT-3 (Ad genome 3497734958). The TaqMAn probe was 5'-CGCCGCCCATGCAACAAGCTT-3' (Ad genome 34930-34951).

\section{Abbreviations}

Ad: Adenovirus; CRAd: Conditionally-Replicative Adenovirus; HR: homologous recombination; MOI: multiplicity of infection; hsp: heat shock protein; MK: midkine; MCS: multiple cloning site; ITR: inverted terminal repeat; TSP: tumor specific promoter.

\section{Competing interests}

The author declares that he has no competing interests.

\section{Authors' contributions}

YSH-designed the cloning strategies, performed the experiments and wrote the manuscript.

\section{Acknowledgements}

This study was performed during a research fellowship at the Division of Human Gene Therapy, University of Alabama at Birmingham, Birmingham, AL, USA and was funded by the by the Birmingham Jewish Federation. The author wishes to thank David T. Curiel, Jerry L. Blackwell, Yasuo Adachi, Hongju Wu and Koichi Takayama for their support, helpful discussions and valuable reagents. Ming Wang performed the real-time PCR. Hui Li is acknowledged for technical support. Current research is funded by the German-Israeli Foundation grant no. 817/2004, Israel Science Foundation grant no. 573/03, the Israeli Ministry of Health, Chief Scientist Office, and the Hadassah Women's health fund.

\section{References}

I. Berkner KL, Sharp PA: Generation of adenovirus by transfection of plasmids. Nucleic Acid Res 1983, I I (17):6003-20.

2. Ruben M, Bacchetti S, Graham FL: Covalently closed circles of adenovirus 5 DNA. Nature 1983, 30I(5896): 172-4.

3. McGrory WJ, Bautista DS, Graham FL: A simple technique for the rescue of early region I mutations into infectious human adenovirus type 5. Virology 1988, 163(2):6I4-7.

4. Bett AJ, Haddara W, Prevec L, Graham FL: An efficient and flexible system for construction of adenovirus vectors with inser- 
tions or deletions in early regions I and 3. PNAS 1994 9I(19):8802-6.

5. $\mathrm{Ng}$ P, Parks RJ, Cummings DT, Evelegh CM, Graham FL: An enhanced system for construction of adenoviral vectors by the two-plasmid rescue method. Hum Gene Ther 2000, I I(5):693-9.

6. He TC, Zhou S, da Costa LT, Yu J, Kinzler KW, Vogelstein B: A simplified system for generating recombinant adenoviruses. PNAS 1998, 95(5):2509-14.

7. Danthinne X, Werth E: New tools for the generation of EI- and/ or E3-substituted adenoviral vectors. Gene Ther 2000 7(I):80-7.

8. Mizuguchi $\mathrm{H}$, Kay MA: Efficient construction of a recombinant adenovirus vector by an improved in vitro ligation method. Hum Gene Ther 1998, 9(17):2577-83.

9. Mizuguchi $\mathrm{H}$, Kay MA: A simple method for constructing EIand EI/E4-deleted recombinant adenoviral vectors. Hum Gene Ther 1999, I0( I 2):2013-7.

10. Souza DW, Armentano D: Novel cloning method for recombinant adenovirus construction in Escherichia coli. Biotechniques 1999, 26(3):502-8.

11. Stow ND: Cloning of a DNA fragment from the left-hand terminus of the adenovirus type 2 genome and its use in sitedirected mutagenesis. J Virol I98I, 37(I): $17 \mid-80$.

12. Haviv YS, Curiel DT: Engineering regulatory elements for conditionally-replicative adenoviruses. Curr Gene Ther 2003, 3(4):357-85.

13. Freytag SO, Rogulski KR, Paielli DL, Gilbert JD, Kim JH: A novel three-pronged approach to kill cancer cells selectively: concomitant viral, double suicide gene, and radiotherapy. Hum Gene Ther 1998, 9(9): I 323-33.

14. Adachi Y, Reynolds PN, Yamamoto M, Wang M, Takayama K, Matsubara S, Muramatsu T, Curiel DT: A midkine promoter-based conditionally replicative adenovirus for treatment of pediatric solid tumors and bone marrow tumor purging. Cancer Res 200I, 6 I (2I):7882-8.

15. Takayama K, Reynolds PN, Adachi Y, Kaliberova L, Uchino J, Nakanishi $Y$, Curiel DT: Vascular endothelial growth factor promoter-based conditionally replicative adenoviruses for pancarcinoma application. Cancer Gene Ther 2007, I 4(I): I05-16.

16. Ahmed A, Thompson J, Emiliusen L, Murphy S, Beauchamp RD, Suzuki K, Alemany R, Harrington K, Vile RG: A conditionally replicating adenovirus targeted to tumor cells through activated RAS P-MAPK-selective mRNA stabilization. Nat Biotechnol 2003 2I(7):77I-7.

17. Bauerschmitz G], Guse K, Kanerva A, Menzel A, Herrmann I, Desmond RA, Yamamoto M, Nettelbeck DM, Hakkarainen T, Dall P, Curiel DT, Hemminki A: Triple-targeted oncolytic adenoviruses featuring the cox 2 promoter, EIA transcomplementation, and serotype chimerism for enhanced selectivity for ovarian cancer cells. Mol Ther 2006, I4(2): 164-74.

18. Nettelbeck DM, Rivera AA, Balagué C, Alemany R, Curiel DT: Novel oncolytic adenoviruses targeted to melanoma: specific viral replication and cytolysis by expression of EIA mutants from the tyrosinase enhancer/promoter. Cancer Res 2002, 62(16):4663-70

19. Sauthoff H, Pipiya T, Heitner S, Chen S, Norman RG, Rom WN, Hay JG: Late expression of p53 from a replicating adenovirus improves tumor cell killing and is more tumor cell specific than expression of the adenoviral death protein. Hum Gene Ther 2002, I3(15): 1859-7|.

20. Rohmer S, Mainka A, Knippertz I, Hesse A, Nettelbeck DM: Insulated hsp70B' promoter: stringent heat-inducible activity in replication-deficient, but not replication-competent adenoviruses. J Gene Med 2008, I0(4):340-54.

21. Huang XF, Ren W, Rollins L, Pittman P, Shah M, Shen L, Gu Q, Strube $\mathrm{R}$, Hu F, Chen SY: A broadly applicable, personalized heat shock protein-mediated oncolytic tumor vaccine. Cancer Res 2003, 63(2I):732I-9.

22. Hoffmann D, Wildner O: Efficient generation of double heterologous promoter controlled oncolytic adenovirus vectors by a single homologous recombination step in Escherichia coli. BMC Biotechnol 2006, 6:36.

23. Hernandez-Alcoceba R, Pihalja M, Wicha MS, Clarke MF: A novel, conditionally replicative adenovirus for the treatment of breast cancer that allows controlled replication of Ela- deleted adenoviral vectors. Hum Gene Ther 2000, I I( I4):2009-24.

24. Cuevas $Y$, Hernández-Alcoceba R, Aragones J, Naranjo-Suárez $S$, Castellanos MC, Esteban MA, Martín-Puig S, Landazuri MO, del Peso L: Specific oncolytic effect of a new hypoxia-inducible factordependent replicative adenovirus on von Hippel-Lindaudefective renal cell carcinomas. Cancer Research 2003, 63:6877-6884.

25. Li X, Zhang YP, Kim HS, Bae KH, Stantz KM, Lee SJ, Jung C, Jiménez JA, Gardner TA, Jeng MH, Kao C: Gene Therapy for Prostate Cancer by Controlling Adenovirus Ela and E4 Gene Expression with PSES Enhancer. Cancer Research 2005, 65: I94I-I95I.

26. Li X, Zhang J, Gao H, Vieth E, Bae KH, Zhang YP, Lee SJ, Raikwar S, Gardner TA, Hutchins GD, VanderPutten D, Kao C, Jeng MH: Transcriptional targeting modalities in breast cancer gene therapy using adenovirus vectors controlled-lactalbumin promoter. Mol Cancer Ther 2005, 4:1850-1859.

27. Sarkar D, Su ZZ, Vozhilla N, Park ES, Randolph A, Valerie K, Fisher PB: Targeted virus replication plus immunotherapy eradicates primary and distant pancreatic tumors in nude mice. Cancer Res 2005, 65( (19):9056-63.

28. Sarkar D, Lebedeva IV, Su ZZ, Park ES, Chatman L, Vozhilla N, Dent P, Curiel DT, Fisher PB: Eradication of Therapy-Resistant Human Prostate Tumors Using a Cancer Terminator Virus. Cancer Research 2007, 67:5434-5442.

29. Renaut L, Bernard C, D'Halluin JC: A rapid and easy method for production and selection of recombinant adenovirus genomes. J Virol Methods 2002, I00(I-2):|2|-3|.

30. Ciocca DR, Calderwood SK: Heat shock proteins in cancer: diagnostic, prognostic, predictive, and treatment implications. Cell Stress Chaperones 2005, I 0(2):86-103.

31. Amin J, Fernandez M, Ananthan J, Lis JT, Voellmy R: Cooperative binding of heat shock transcription factor to the Hsp70 promoter in vivo and in vitro. J Biol Chem 1994, 269(7):4804-I I.

32. Rome C, Couillaud F, Moonen CT: Spatial and temporal control of expression of therapeutic genes using heat shock protein promoters. Methods 2005, 35(2): 188-98.

33. Lee YJ, Galoforo SS, Battle P, Lee H, Corry PM, Jessup JM: Replicating adenoviral vector-mediated transfer of a heat-inducible double suicide gene for gene therapy. Cancer Gene Ther 200 I, 8(6):397-404

34. Haviv YS, Blackwell JL, Li H, Wang M, Lei X, Curiel DT: Heat shock and heat shock protein $70 \mathrm{i}$ enhance the oncolytic effect of replicative adenovirus. Cancer Res 200I, 6 I(23):836I-5.

35. Bischoff JR, Kirn DH, Williams A, Heise C, Horn S, Muna M, Ng L, Nye JA, Sampson-Johannes A, Fattaey A, McCormick F: An adenovirus mutant that replicates selectively in p53-deficient human tumor cells. Science 1996, 274(5286):373-6.

36. O'Shea CC, Johnson L, Bagus B, Choi S, Nicholas C, Shen A, Boyle L, Pandey K, Soria C, Kunich J, Shen Y, Habets G, Ginzinger D, McCormick F: Late viral RNA export, rather than p53 inactivation, determines ONYX-0I 5 tumor selectivity. Cancer Cell 2005, 6(6):6||-623.

Publish with Bio Med Central and every scientist can read your work free of charge

"BioMed Central will be the most significant development for disseminating the results of biomedical research in our lifetime. "

Sir Paul Nurse, Cancer Research UK

Your research papers will be:

- available free of charge to the entire biomedical community

- peer reviewed and published immediately upon acceptance

- cited in PubMed and archived on PubMed Central

- yours - you keep the copyright 